\title{
Lifetimes of unoccupied surface states on $\operatorname{Pd}(111)$
}

\author{
A. Schäfer, I. L. Shumay, M. Wiets, M. Weinelt, and Th. Fauster \\ Lehrstuhl für Festkörperphysik, Universität Erlangen-Nürnberg, Staudtstrasse 7, D-91058 Erlangen, Germany \\ E. V. Chulkov, V. M. Silkin, and P. M. Echenique \\ Departamento de Física de Materiales, Facultad de Ciencias Químicas, Euskal Herriko Unibertsitatea, Donostia International Physics \\ Center (DIPC) and Centro Mixto CSIC-UPV/EHU, 20018 San Sebastián, Basque Country, Spain
}

(Received 2 November 1999)

\begin{abstract}
Using time-resolved two-photon photoemission we have measured the linewidths and lifetimes of the unoccupied Shockley surface state and first image-potential state on $\operatorname{Pd}(111)$. Calculated values are in good agreement with the experimental results.
\end{abstract}

\section{INTRODUCTION}

Lifetimes and linewidths of surface states have been of increasing interest in recent years due to technological advances in experiment and progress in theoretical models. The focus on surface states stems from the surface sensitivity of photoelectron spectroscopy, ${ }^{1}$ but similar results are also obtained for hot electrons in bulk bands. ${ }^{2}$ Improved resolution and sensitivity of photoelectron experiments permits an accurate measurement of the linewidth $\Gamma$ of surface states. ${ }^{3}$ The availability of femtosecond lasers opened up the possibility of measuring the lifetimes $\tau$ of unoccupied surface states with matching resolution. ${ }^{4}$ In a simple picture these quantities should be related by $\Gamma \tau=\hbar$. Dephasing processes, such as quasielastic scattering processes, influence only the phase of the wave function and not the population of the state. Therefore, the linewidth is usually larger than expected from the above relation. ${ }^{5}$ For occupied surface states the lifetime cannot be measured directly and can be obtained only by minimizing the quasielastic scattering processes. This can be done to some extent by extrapolation to zero temperature ${ }^{6}$ or zero defects. ${ }^{7}$ However, the sample quality in particular sets unsurpassable limits on this extrapolation.

The theoretical models usually assume an ideal defectfree surface at zero temperature and the results have to be compared to the measured lifetimes. The surface states studied in detail are mostly occupied states ${ }^{1}$ and, therefore, experimental information on the lifetime is not available. Notable exceptions are image-potential states for which rather good agreement between theory and experiment has been found. ${ }^{4,8}$ In order to extend these studies to the Shockleytype surface states found for $s p$ band gaps, we studied the $\operatorname{Pd}(111)$ surface. On this surface an unoccupied surface state exists. ${ }^{9}$ The minimum of the parabola of this free-electronlike band is $1.26 \mathrm{eV}$ above the Fermi energy $E_{F}$ at the center of the surface Brillouin zone. ${ }^{9,10}$ On most other close-packed surfaces this band is partially occupied and this difference accounts for the variety of the chemical reactivities of different noble metal surfaces. ${ }^{11}$

In the following we present first the experimental setup and the results for the Shockley-type surface states and the lowest image-potential state. The next section outlines the theoretical model and gives the corresponding values for the
$\operatorname{Pd}(111)$ surface. The final section compares and discusses the experimental and theoretical results.

\section{EXPERIMENT}

The measurement of linewidths and lifetimes of unoccupied states is done by time-resolved two-photon photoelectron spectroscopy (2PPE). ${ }^{4}$ A first laser pulse excites an electron from the metal into an intermediate state above the Fermi level. A second photon lifts the electron above the vacuum level $E_{v a c}$ so it may leave the surface and can be detected in an energy analyzer. Scanning the spectrum with the analyzer yields the energy and linewidth of the state. Varying the time delay between the two laser pulses at fixed analyzer energy shows the decay of the population in the intermediate state. This permits the determination of lifetimes in the time domain with femtosecond resolution.

\section{A. Experimental setup}

The experimental arrangement for 2PPE used an ultrahigh-vacuum (UHV) system for sample preparation and electron energy analysis. The sample preparation followed the procedures described before. ${ }^{10,12,13}$ For the 2PPE measurements the sample was held at $150{ }^{\circ} \mathrm{C}$ to avoid hydrogen adsorption. Electrons were detected parallel to the surface normal after an electrostatic hemispherical analyzer (Omicron EA $125 \mathrm{HR}$ ) with an acceptance cone of $\pm 1.0^{\circ}$. The energy resolution was tuned to $30 \mathrm{meV}$.

The laser setup consists of a home-built Ti:sapphire laser $^{14}$ pumped by a $5 \mathrm{~W}$ diode-pumped solid-state laser (Spectra Physics, Millennia). The IR pulse length at $790 \mathrm{~nm}$ is $12 \mathrm{fs}$ at a repetition rate of $87 \mathrm{MHz}$. Introducing a slit in the cavity increases the pulse length to $23 \mathrm{fs}$, but allows wavelength tuning between 750 and $820 \mathrm{~nm}$. Ten percent of the output power of $560 \mathrm{~mW}$ was sent after pulse compression directly to the sample. The major part was used to generate the third harmonic of the IR radiation. The power of the UV beam at $263 \mathrm{~nm}(253 \mathrm{~nm})$ was $10 \mathrm{~mW}(2.5 \mathrm{~mW})$. After pulse compression the cross-correlation between the UV and IR beams was measured by monitoring the 2PPE signal from the occupied surface state of a $\mathrm{Cu}(111)$ sample. ${ }^{15}$ The crosscorrelation of 46 fs constitutes an improvement by a factor of 


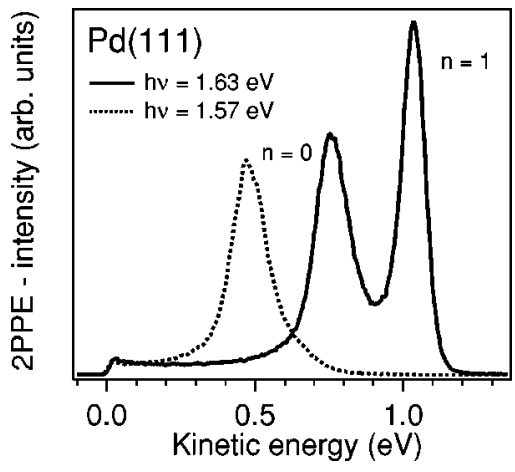

FIG. 1. Two-photon photoemission spectra for $\operatorname{Pd}(111)$ at $h \nu$ $=1.57 \mathrm{eV}$ (dashed curve) and $1.63 \mathrm{eV}$ (solid line). The spectra are plotted as a function of the kinetic energy with respect to the $\operatorname{Pd}(111)$ sample. At the lower photon energy only the $n=0$ Shockley state is seen, whereas at the higher photon energy the $n=1$ image-potential state appears.

2 over previous experiments ${ }^{4,15,16}$ and corresponds to a pulse length of $40 \mathrm{fs}$ for the UV beam. Using the longer IR pulses increased the conversion efficiency of the frequency tripler without any significant increase of the cross-correlation width compared to shorter IR pulses. The angle of incidence at the sample of the $p$-polarized light for the two collinear beams was $45^{\circ}$ relative to the surface normal.

\section{B. Energy-resolved spectra}

Figure 1 shows 2PPE spectra from a $\mathrm{Pd}(111)$ surface measured at 760 and $790 \mathrm{~nm}$ IR wavelength. The longer wavelength corresponds to a photon energy of $h \nu=1.57 \mathrm{eV}$ and shows only the Shockley surface state. At the higher photon energy of $h \nu=1.63 \mathrm{eV}$ the $n=1$ image state can be seen as well. From the low-energy cutoff the work function of the clean $\mathrm{Pd}(111)$ sample is determined as $5.50 \pm 0.01 \mathrm{eV}$, which is within the range of previously reported values of $5.6 \mathrm{eV},{ }^{9} 5.55 \mathrm{eV},{ }^{17}$ and $5.44 \pm 0.03 \mathrm{eV} .{ }^{10}$ The wavelength dependence of the energies of the states and the timeresolved data (see Sec. II C) show that the Shockley surface state is excited by a photon of energy $h \nu$ and the electron is then emitted by $3 h \nu$ photon. For the image-potential state the reverse order applies. Using this information we obtain the energies of the Shockley and image-potential states to be $1.35 \pm 0.02 \mathrm{eV}$ and $4.90 \pm 0.03 \mathrm{eV}$ above $E_{F}$, respectively. The first value is somewhat higher than the previous results of $1.3 \mathrm{eV}$ (Ref. 9) and $1.26 \pm 0.04 \mathrm{eV} .{ }^{10}$ However, these data were obtained at room temperature whereas in our experiments the sample was held at $150^{\circ} \mathrm{C}$. A downward shift of the Shockley-state energy of $0.11 \mathrm{eV}$ upon cooling the sample from room temperature to $85 \mathrm{~K}$ has been reported, ${ }^{13}$ which is consistent with the upward shift during heating observed here. The energy of the image-potential state is 4.90 $\pm 0.03 \mathrm{eV}$, in excellent agreement with previous measurements by $2 \mathrm{PPE},{ }^{10,17}$ but somewhat smaller than the inverse photoemission result of $5.1 \mathrm{eV}$ obtained at lower resolution. ${ }^{9}$

The dispersion of the surface states can be described well by a free-electron-like behavior characterized by effective masses of $(0.2 \pm 0.05) m_{e}$ and $(1.0 \pm 0.1) m_{e}$ for the Shockley and image-potential states, respectively. The first value is significantly lower than the result of $0.3 m_{e}$ from the inverse photoemission study, ${ }^{9}$ but in good agreement with the calculated value of $0.22 m_{e} \cdot{ }^{18}$

The last piece of information that can be extracted from energy-resolved spectra as shown in Fig. 1 is the linewidth of the peaks. A careful lineshape analysis (from spectra taken at sufficient delay ${ }^{4,15}$ ) yields values for the intrinsic linewidth $\Gamma$ of $100 \pm 10 \mathrm{meV}$ and $32 \pm 4 \mathrm{meV}$ for the Shockley and image-potential states, respectively. These numbers are a factor of 2 smaller than those reported previously. ${ }^{10}$ The measurement times in the earlier work were about a factor of 10 longer than in the current study. This could have resulted in some hydrogen adsorption from the residual gas. At room temperature we observe a reduction by a factor of 3 of the intensity and a 50\% increase of the linewidth of the Shockley state within ten minutes at $3.5 \times 10^{-11}$ mbar partial pressure of $\mathrm{H}_{2}$. The image-potential state loses intensity on a time scale that is a factor of 2 larger than the corresponding time scale for the Shockley state.

At the photon energy used here, the Shockley state could be resonantly excited from the occupied surface state at $E_{F}$ $-0.3 \mathrm{eV} .{ }^{19}$ The previous $2 \mathrm{PPE}$ study used a larger photon energy for the first excitation step and the Shockley state was observed with much smaller intensity than the imagepotential state. ${ }^{10}$ In the present work the sensitivity to hydrogen adsorption could be attributed therefore to the Shockley state or the occupied surface state. Measurements using the UV light in both excitation steps revealed no increased sensitivity to hydrogen adsorption compared to experiments with excitation using IR light. The low intensity and large linewidth of the Shockley state observed in the earlier studies $^{9,10}$ can be attributed most likely to the longer measurement times at room temperature and, consequently, larger hydrogen contamination.

The Shockley state of $\Lambda_{1}$ symmetry was also observed using $s$-polarized IR light. According to the dipole selection rules ${ }^{20}$ this is possible only from initial states of $\Lambda_{3}$ symmetry. In the relevant energy range, bulk bands ${ }^{21}$ as well as the occupied surface state ${ }^{19,22}$ possess this symmetry and could serve as initial states. A resonant excitation from the occupied surface state should lead to a reduced linewidth compared to off-resonant excitation., ${ }^{4,23}$ A linewidth narrowing compared to the off-resonant excitation by UV light was not observed. In conclusion, we have no evidence for an excitation of the Shockley state from the occupied surface state at photon energies around $1.6 \mathrm{eV}$. To resolve this point, further studies covering a larger photon-energy range are needed.

\section{Time-resolved spectroscopy}

For the direct experimental determination of the decay of the population in the unoccupied states we tune the energy analyzer to the desired energy and measure the signal as a function of the delay between the two laser pulses. Data for the two peaks visible in the $h \nu=1.63 \mathrm{eV}$ spectrum of Fig. 1 are shown after background subtraction on a semilogarithmic scale in Fig. 2. The maximum of the cross-correlation curve shown by solid dots corresponds to the maximum overlap between the laser pulses. This curve was obtained for the occupied surface state of a $\mathrm{Cu}(111)$ sample $^{15}$ and defines the zero on the time scale. The cross-correlation was recorded 


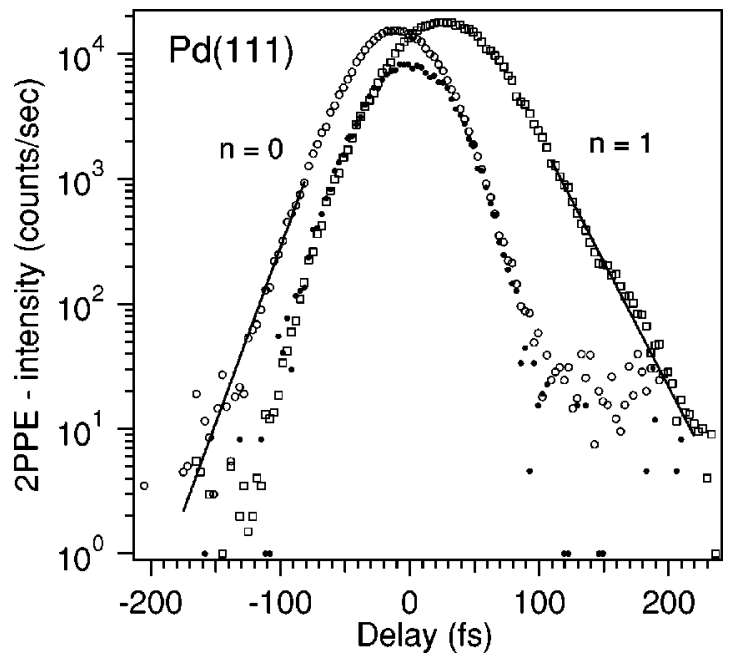

FIG. 2. Time-resolved spectra for the Shockley (open circles, $n=0)$ and image-potential states (open squares, $n=1$ ) on $\operatorname{Pd}(111)$. The solid dots show the cross-correlation determined for the occupied Shockley state on $\mathrm{Cu}(111)$ (Ref. 15).

before and after the measurements on the Pd(111) sample and showed drifts below 1 fs. The curve for the imagepotential state is shifted toward positive delays in Fig. 2, which corresponds to the $h \nu$ light arriving after the $3 h \nu$ light at the sample. The reverse situation is found for the Shockley state and proves the excitation order confirmed in Sec. II B by the photon-energy dependence of the kinetic energy of the peaks. Evaluation of the experimental data following the procedures described in Ref. 4 yields lifetimes $\tau$ of $13 \pm 3$ fs and $25 \pm 4 \mathrm{fs}$ for the Shockley and image-potential states, respectively. These values are in agreement with the straight line fits indicated in Fig. 2, which show a significantly slower decay than the cross-correlation curve. The corresponding decay rates $\hbar / \tau$ of $54 \pm 13 \mathrm{meV}$ and $27 \pm 4 \mathrm{meV}$ are significantly smaller than the intrinsic linewidths reported in Sec. II B. This indicates a significant contribution of dephasing processes to the linewidth. Such processes could be quasielastic scattering by phonons ${ }^{24}$ due to the elevated sample temperature or by residual defects on the surface. ${ }^{25}$

\section{THEORY}

It is well known that the charge density of the $s-p_{z}$ Shockley surface state on metal surfaces located inside the energy gap has a relatively small variation in a plane parallel to the surface. ${ }^{26-30}$ In this particular aspect this surface state is closer to image-potential states ${ }^{31,32}$ than to other symmetry surface states. So with a reasonable accuracy the $s-p_{z}$ surface states can be treated by using a model potential that varies in the $z$ direction (i.e., perpendicular to the surface) and is constant in the plane parallel to the surface. The model potential reproduces the energy gap and binding energy of the surface state and the first image-potential state at the $\Gamma$ point. ${ }^{33,34}$ With this potential, one-electron wave functions $\Psi_{n \mathbf{k}_{\|}}(\mathbf{r})$ $=L^{-1} e^{i \mathbf{k} \|} \cdot \mathbf{r}_{\|} \phi_{n}(z)$ and energies $E_{n \mathbf{k}_{\|}}=E_{n}+\hbar^{2} \mathbf{k}_{\|}^{2} / 2 m$ are obtained within a thin-film model, and then they are used to calculate the damping rate of the surface and image-potential states.

The inverse lifetime $\tau^{-1}=\Gamma / \hbar$ of a quasiparticle can be evaluated as the projection of the imaginary part of the quasiparticle selfenergy $\Sigma\left(\mathbf{r}, \mathbf{r}^{\prime} ; E_{0}\right)$ over the state itself. In the $G W$ approximation only the first term of the expansion of the self-energy in the screened Coulomb interaction is taken into account. ${ }^{35}$ After replacing the Green function by its zero order approximation, the linewidth of the surface state is given by

$$
\begin{aligned}
\Gamma= & -2 / L^{2} \sum_{E_{F}<E_{n \mathbf{k}_{\|}}<E_{0}, \mathbf{q}_{\|}} \int d z \int d z^{\prime} \Phi_{0, n \mathbf{k}_{\|}}\left(z, \mathbf{q}_{\|}\right) \\
& \times \operatorname{Im} W^{\text {ind }}\left(z, z^{\prime} ; \mathbf{q}_{\|} ; E_{0}-E_{n \mathbf{k}_{\|}}\right) \Phi_{0, n \mathbf{k}_{\|}}^{*}\left(z^{\prime}, \mathbf{q}_{\|}\right),
\end{aligned}
$$

where the sums over $\mathbf{q}_{\|}$and $E_{n \mathbf{k}_{\|}}$include, respectively, all vectors from the two-dimensional momentum space and all states lying in energy between the surface (image-potential) state energy $E_{0}$ and the Fermi level $E_{F} ; \Phi_{0, n \mathbf{k}}(z, \mathbf{q} \|)$ is given by

$$
\Phi_{0, n \mathbf{k}_{\|}}\left(z, \mathbf{q}_{\|}\right)=\int d \mathbf{r}_{\|} e^{i \mathbf{q} \|} \cdot \mathbf{r}_{\|} \Psi_{0}^{*}(\mathbf{r}) \Psi_{n \mathbf{k}_{\|}}(\mathbf{r}),
$$

where $\Psi_{0}(\mathbf{r})$ is the surface (image-potential) state wave function. The imaginary part of the screened interaction $\operatorname{Im} W^{\text {ind }}$ satisfies the equation

$$
\begin{aligned}
\operatorname{Im} W^{\text {ind }}\left(z, z^{\prime} ; \mathbf{k}_{\|}, E\right)= & \int d z_{1} \int d z_{2} \\
& \times V\left(z, z_{1} ; \mathbf{k}_{\|}\right) \operatorname{Im} \chi\left(z_{1}, z_{2} ; \mathbf{k}_{\|}, E\right) \\
& \times V\left(z_{2}, z^{\prime} ; \mathbf{k}_{\|}\right) .
\end{aligned}
$$

Here $V\left(z, z^{\prime} ; \mathbf{k}_{\|}\right)$and $\chi\left(z, z^{\prime} ; \mathbf{k}_{\|}, E\right)$ are two-dimensional Fourier transforms of the bare Coulomb interaction and the density response function, respectively. Within the random phase approximation $\chi\left(z, z^{\prime} ; \mathbf{k}_{\|}, E\right)$ is evaluated with the use of the density response function of noninteracting electrons, $\chi^{0}$, which is calculated in terms of both eigenfunctions $\phi_{n}(z)$ and eigenvalues $E_{n}$ of the one-electron Hamiltonian. The results presented below have been found to be well converged and they have been obtained for 30-50 layer films by summing in $\chi^{0}$ over all one-electron states up to an energy of $100-130 \mathrm{eV}$ above the vacuum level. These wave functions and energies have been obtained as solutions of the onedimensional Schrödinger equation with the model potential of Refs. 33 and 34, which approaches, far outside the surface, the classical image potential. As was shown in Refs. 33 and 34, this model potential gives wave functions of the surface and image-potential states in excellent agreement with those obtained from first-principles calculations. The method briefly described above has been used in Refs. 8 and 36 to calculate the lifetime of image-potential states on $\mathrm{Cu}(100)$ and $\mathrm{Cu}(111)$. The evaluated lifetimes have been found to be in good agreement with time-resolved 2PPE measurement results. ${ }^{4,16}$

To take into account the surface corrugation we calculate the lifetime of the surface states by using experimental values of the effective mass of these states and first-principles bulk calculation results for bands forming the bottom of the energy gap. So the sum over $E_{n \mathbf{k}_{\|}}$in Eq. (1) includes the 
TABLE I. Energies relative to $E_{F}$, linewidths, and inverse lifetimes for the Shockley $(n=0)$ and $n=1$ image-potential states on $\operatorname{Pd}(111)$. The last columns give the work function $\Phi$ and the appropriate references. For binding energies and further references, see also Ref. 34.

\begin{tabular}{lccccccc}
\hline \hline $\begin{array}{l}E_{0} \\
(\mathrm{eV})\end{array}$ & $\begin{array}{c}\Gamma_{0} \\
(\mathrm{meV})\end{array}$ & $\begin{array}{c}\hbar / \tau_{0} \\
(\mathrm{meV})\end{array}$ & $\begin{array}{c}E_{1} \\
(\mathrm{eV})\end{array}$ & $\begin{array}{c}\Gamma_{1} \\
(\mathrm{meV})\end{array}$ & $\begin{array}{c}\hbar / \tau_{1} \\
(\mathrm{meV})\end{array}$ & $\begin{array}{c}\Phi \\
(\mathrm{eV})\end{array}$ & Reference \\
\hline 1.35 & 100 & 54 & 4.90 & 32 & 27 & 5.50 & this expt. \\
1.35 & & 37 & 4.90 & & 30 & 5.50 & calc. \\
1.26 & 200 & & 4.89 & 70 & & 5.44 & Ref. 10 \\
1.26 & & 36 & 4.89 & & 27 & 5.44 & calc. \\
1.3 & & & 5.1 & & & 5.6 & $\begin{array}{c}\text { Ref. } 9 \\
\text { Ref. 17 } \\
1.1\end{array}$ \\
0.9 & & 4.9 & & & 5.55 & $\begin{array}{c}\text { Ref. 37 } \\
\text { Ref. 18 }\end{array}$ \\
\hline \hline
\end{tabular}

dispersion curves $E_{n \mathbf{k}}=E_{n}+\hbar^{2} \mathbf{k}_{\|}^{2} / 2 m_{n}^{*}$, where $m_{n}^{*}$ is the effective mass of the $n$th state in units of the free-electron mass.

Two-photon photoemission experiments on $\operatorname{Pd}(111)$ have been performed at $150{ }^{\circ} \mathrm{C}$ (this work) and room temperature (Ref. 10), respectively. These experiments gave slightly different binding energies of the surface states as well as the work function (see Table I). So we have performed two calculations to obtain the inverse lifetime for these two sets of surface states. The model potential describing the binding energies of surface states found at room temperature has been taken from Ref. 34. We have obtained another set of bulk potential parameters, which reproduces experimental energies of the surface states at $150{ }^{\circ} \mathrm{C}$. The results of the calculation are listed in the second and fourth rows of Table I.

\section{DISCUSSION}

The agreement between experimental data and theoretical calculations in Table I is reasonably good considering the complexity of the methods involved on both sides. The available information gives energies around $1.3 \mathrm{eV}$ and $4.9 \mathrm{eV}$ for the Shockley and image-potential states, respectively. The work function has a value around $5.5 \mathrm{eV}$ and shows a somewhat larger scatter. This might be attributed to the sensitivity to the surface preparation and the surface potential on the experimental and theoretical sides, respectively. For example, local density calculations ${ }^{18,38,39}$ give work functions of $5.5-5.7 \mathrm{eV}$, while a many-body calculation, ${ }^{18}$ which re- produces correctly the image potential in vacuum, gives $\Phi$ $=6.18 \mathrm{eV}$. This high work function automatically leads to a very high energy of the image-potential state relative to $E_{F}$. The second image-potential state was not accessible in this work, but the binding energy of $0.15 \mathrm{eV}$ (Ref. 10) is in excellent agreement with theoretical calculations. ${ }^{34}$

The experimental decay rates $\hbar / \tau$ are upper limits, because contributions from residual defects and from the finite sample temperature cannot be excluded. ${ }^{6,7}$ Nevertheless, the agreement with the calculated values is good. Experimentally, the decay rate for the Shockley state is found to be a factor of 2 larger than the corresponding value for the first image-potential state in experiment. This ratio is found to be significantly smaller in the calculations. A possible explanation could be the stronger sensitivity to defects and adsorbates of the Shockley state compared to the image-potential state, which has a wave function extended further away from the surface. In this context we mention that the decay rate for the second image-potential state is calculated to be $7.4 \mathrm{meV}$. An experimental value is not available, but the ratio of lifetimes between $n=2$ and $n=1$ states is found to be around 4 for other surfaces. ${ }^{40}$

On the theoretical side, the relatively low decay rate for the $n=0$ state may be attributed to the absence of $d$ states in our model. It is well known that bulk Pd is characterized by a high density of $d$ states at the Fermi level. ${ }^{21}$ Unoccupied $d$ states lie in a narrow energy interval of $0.3 \mathrm{eV}$ just above $E_{F}$ and may participate as final states in the decay of both surface states. Because of the small penetration of imagepotential states into the bulk [for $\operatorname{Pd}(111)$ the penetration of the first image-potential state is $4.6 \%$ (Ref. 34)] one can expect only a small impact of $d$ states on the decay rate of image-potential states. At the same time, $d$ states may have a more profound effect on the decay rate of the Shockley state by increasing the linewidth of this state.

A significant difference between linewidth and decay rate for the Shockley state is observed directly by our experiments. This shows that one has to be careful in using linewidths of occupied Shockley states on other surfaces ${ }^{1}$ as information for the lifetime of these hole states. Instead, a careful extrapolation to zero temperature ${ }^{6}$ or zero defects ${ }^{7}$ has to be done. Only for unoccupied states do time-resolved two-photon photoemission studies permit unambiguous separation between energy and phase decay.

\section{ACKNOWLEDGMENTS}

This work was supported by the Departamento de Educación del Gobierno Vasco and by Acciones Integradas Hispano-Alemanas.
${ }^{1}$ R. Matzdorf, G. Meister, and A. Goldmann, Surf. Sci. 286, 56 (1993).

${ }^{2}$ E. Knoesel, A. Hotzel, and M. Wolf, Phys. Rev. B 57, 12812 (1998).

${ }^{3}$ D. Purdie, M. Hengsberger, M. Garnier, and Y. Baer, Surf. Sci. 407, L671 (1998).

${ }^{4}$ I. L. Shumay, U. Höfer, Ch. Reuß, U. Thomann, W. Wallauer, and Th. Fauster, Phys. Rev. B 58, 13974 (1998).
${ }^{5}$ Ch. Reuß, I. L. Shumay, U. Thomann, M. Kutschera, M. Weinelt, Th. Fauster, and U. Höfer, Phys. Rev. Lett. 82, 153 (1999).

${ }^{6}$ R. Matzdorf, G. Meister, and A. Goldmann, Phys. Rev. B 54, 14 807 (1996)

${ }^{7}$ F. Theilmann, R. Matzdorf, G. Meister, and A. Goldmann, Phys. Rev. B 56, 3632 (1997).

${ }^{8}$ E. V. Chulkov, I. Sarria, V. M. Silkin, J. M. Pitarke, and P. M. Echenique, Phys. Rev. Lett. 80, 4947 (1998). 
${ }^{9}$ S. L. Hulbert, P. D. Johnson, and M. Weinert, Phys. Rev. B 34, 3670 (1986).

${ }^{10}$ R. Fischer, S. Schuppler, N. Fischer, Th. Fauster, and W. Steinmann, Phys. Rev. Lett. 70, 654 (1993).

${ }^{11}$ N. Memmel and E. Bertel, Phys. Rev. Lett. 75, 485 (1995).

${ }^{12}$ B. Eisenhut, J. Stober, G. Rangelov, and Th. Fauster, Phys. Rev. B 47, 12980 (1993).

${ }^{13}$ W. Wallauer, R. Fischer, and Th. Fauster, Surf. Sci. 364, 297 (1996).

${ }^{14}$ M. T. Asaki, C.-P. Huang, D. Garvey, J. Zhou, H. C. Kapteyn, and M. M. Murnane, Opt. Lett. 18, 977 (1993).

${ }^{15}$ T. Hertel, E. Knoesel, A. Hotzel, M. Wolf, and G. Ertl, J. Vac. Sci. Technol. A 15, 1503 (1997).

${ }^{16}$ U. Höfer, I. L. Shumay, Ch. Reuß, U. Thomann, W. Wallauer, and Th. Fauster, Science 277, 1480 (1997).

${ }^{17}$ G. D. Kubiak, J. Vac. Sci. Technol. A 5, 731 (1987).

${ }^{18}$ M. Heinrichsmeier, A. Fleszar, W. Hanke, and A. G. Eguiluz, Phys. Rev. B 57, 14974 (1998).

${ }^{19}$ W. Eberhardt, S. G. Louie, and E. W. Plummer, Phys. Rev. B 28, 465 (1983).

${ }^{20}$ W. Eberhardt and F. J. Himpsel, Phys. Rev. B 21, 5572 (1980); 23, 5650 (E) (1981).

${ }^{21}$ D. A. Papaconstantopoulos, Handbook of the Band Structure of Elemental Solids (Plenum Press, New York, 1986).

${ }^{22}$ O. Bisi and C. Calandra, Surf. Sci. 83, 83 (1979).

${ }^{23}$ W. Wallauer and Th. Fauster, Surf. Sci. 374, 44 (1997).

${ }^{24}$ E. Knoesel, A. Hotzel, and M. Wolf, J. Electron Spectrosc. Relat. Phenom. 88-91, 577 (1998).
${ }^{25}$ Th. Fauster, Ch. Reuß, I. L. Shumay, and M. Weinelt, Chem. Phys. 251, 111 (2000).

${ }^{26}$ S. G. Louie, Phys. Rev. Lett. 40, 1525 (1978).

${ }^{27}$ L. Ley, G. P. Kerker, and N. Martensson, Phys. Rev. B 23, 2710 (1981).

${ }^{28}$ E. V. Chulkov, V. M. Silkin, and E. N. Shirykalov, Surf. Sci. 188, 287 (1987).

${ }^{29}$ J. Inglesfield and G. A. Benesh, Phys. Rev. B 37, 6682 (1988).

${ }^{30}$ E. V. Chulkov and V. M. Silkin, Surf. Sci. 215, 385 (1989).

${ }^{31}$ P. M. Echenique and J. B. Pendry, J. Phys. C 11, 2065 (1978).

${ }^{32}$ P. M. Echenique and J. B. Pendry, Prog. Surf. Sci. 32, 111 (1990).

${ }^{33}$ E. V. Chulkov, V. M. Silkin, and P. M. Echenique, Surf. Sci. 391, L1217 (1997).

${ }^{34}$ E. V. Chulkov, V. M. Silkin, and P. M. Echenique, Surf. Sci. 437, 330 (1999).

${ }^{35}$ L. Hedin and S. Lundqvist, Solid State Phys. 23, 1 (1969).

${ }^{36}$ I. Sarrfia, J. Osma, E. V. Chulkov, J. M. Pitarke, and P. M. Echenique, Phys. Rev. B 60, 11795 (1999).

${ }^{37}$ W. Dong, G. Kresse, J. Furthmüller, and J. Hafner, Phys. Rev. B 54, 2157 (1996).

${ }^{38}$ M. Methfessel, D. Hennig, and M. Scheffler, Phys. Rev. B 46, 4816 (1992).

${ }^{39}$ V. Pallassana, M. Neurock, L. B. Hansen, B. Hammer, and J. K. Norskov, Phys. Rev. B 60, 6146 (1999).

${ }^{40}$ Th. Fauster and W. Steinmann, in Photonic Probes of Surfaces, Vol. 2 of Electromagnetic Waves: Recent Developments in Research, edited by P. Halevi (North-Holland, Amsterdam, 1995), Chap. 8, p. 347. 